\title{
An introduction to the Higgs mechanism based on classical physics secondary school curriculum
}

\section{Giovanni Organtini*}

Dip. di Fisica Sapienza Università di Roma \& INFN-Sez. di Roma

E-mail: giovanni.organtiniduniromal.it

The Higgs mechanism is introduced in a completely classical framework in which the concept of energy is reviewed. After a brief review of the classical energy of a particle in a gravitational or electromagnetic fields, we show that postulating the existence of a new field (the Higgs field) one can easily introduce a new term in the energy that is consistent with the relativistic energy at rest of a particle, making the mass of a particle the result of a dynamic effect of the interaction with a scalar field. Our model also gives rise to interaction terms between particles and the new field, as well as to the mass of such a field (i.e. to the Higgs boson).

Our approach allows a formal introduction of the Higgs field dynamics, much similar to other topics of classical textbooks, that does not require any knowledge of quantum field theory.

EPS-HEP 2017 - Venezia, Italy 5 - 12 July 2017

\footnotetext{
*Speaker.
} 


\section{Introduction}

The way in which modern physics is introduced in textbooks is usually different with respect to classical physics topics. The reason for that is that it is difficult to find a relatively easy formulation of modern theories, while consolidated ones have found, with time, a solid mathematical base at every student's reach. Therefore, for students, modern physics appears to be different with respect to classical physics. The latter is taught with a formal approach, while the first consists just in a collection of qualitative statements and rules with no formal justification.

In this presentation we suggest a new introduction to the Higgs mechanism based on concepts that any secondary school students is supposed to know, not involving any quantum field theory.

\section{Energy revised}

The energy [四] of a particle of mass $m$ in a gravitational field $\mathbf{G}$ can be written as $E_{G}=m U_{G}$, where $U_{G}=\int \mathbf{G} \cdot d \mathbf{x}$, called the gravitational potential, is a scalar function of the field $\mathbf{G}$ and of coordinates $\mathbf{x}$. Similarly, if the particle has an electric charge $q$, its energy in an electrostatic field $\mathbf{E}$ can be written as $E_{V}=q V$, where $V$ is again a scalar function of the field and the coordinates [四]. Even in the case of a particle with magnetic dipole moment $\boldsymbol{\mu}=I \mathbf{S}, \mathbf{S}$ being a vector perpendicular to the plane of a current loop $I$, whose magnitude is equal to the area of the enclosed surface, we can write the energy as $E_{B}=-\boldsymbol{\mu} \cdot \mathbf{B}=-I U_{B}$ when interacting with a magnetic field $\mathbf{B}$. In this case, $U_{B}=-\int \mathbf{B} \cdot d \mathbf{S}$ is still a scalar function of the field and the coordinates. In general, then, the energy of a particle in a field can be written as $c_{i} U_{i}$ where $c_{i}$ is a coupling constant (the mass, the electric charge, the current) and $U_{i}$ a scalar function of the field $\mathbf{F}_{i}$ with which the particle interacts, and the coordinates. We can call that function the potential, even if such a term is not normally used in the case of the magnetic field.

If the particle is contained in a volume $L^{3}$, one can measure the energy contained in that volume as the energy of the particle plus the energy of the fields contained in it. Electromagnetic fields contributes to such an energy as $E_{E E}+E_{B B}=L^{3}\left(\frac{\varepsilon_{0}}{2} E^{2}+\frac{1}{2 \mu_{0}} B^{2}\right)$. In principle one can add a contribution for the gravitational field $E_{G G}=L^{3} \frac{\gamma}{2} G^{2}$, but, as a matter of fact, $\gamma=0$. One can think to these terms as arising from the auto-interaction of the fields. In general, the energy density due to the field $\mathbf{F}_{i}$ contained in a volume is of the form $E_{F F} L^{-3}=\frac{f_{i}}{2} F^{2}$.

As a general rule, in classical physics the energy contained in a volume $L^{3}$ filled with fields and one particle can be written as $E=\sum_{i} c_{i} U_{i}+L^{3} \sum_{i} \frac{f_{i}}{2} F^{2}$. Including special relativity [ $\square$ ], a new term appears in the sum of the form $m c^{2}$. Such a term is nor in the form $c_{i} U_{i}$ nor in the form $\frac{f_{i}}{2} F^{2}$, breaking such a nice symmetry according to which the energy in a volume is a measure of the strength of interactions between particles and fields and fields with themselves.

\section{The Higgs field}

One can recover the symmetry assuming that a new field $\mathscr{H}$ exists, whose potential is $U_{\mathscr{H}}$, with which every particle interacts. We chosen a scalar field as the simplest possible one and we call this the Higgs field. In this case one must add terms of the form $c_{H} U_{\mathscr{H}}+L^{3} \frac{f_{H}}{2} \mathscr{H}^{2}$ to the energy. Unlike classical fields, for which energy goes to zero when the field goes to zero, for the field $\mathscr{H}$ 
the minimum possible energy is attained when $\mathscr{H}=H_{0} \neq 0$. We can then write $\mathscr{H}=H_{0}+H$ and accordingly $U_{\mathscr{H}}=U_{H}^{0}+U_{H}$. The extra contribution to the energy becomes then

$$
E_{H}=c_{H}\left(U_{H}^{0}+U_{H}\right)+L^{3} \frac{f_{H}}{2}\left(H_{0}+H\right)^{2}=c_{H} U_{H}^{0}+c_{H} U_{H}+L^{3} \frac{f_{H}}{2} H_{0}^{2}+L^{3} \frac{f_{H}}{2} H^{2}+L^{3} f_{H} H_{0} H
$$

The first term $c_{H} U_{H}^{0}$ is a constant that depends on the coupling constant $c_{H}$ between the particle and the $\mathscr{H}$ field. It then depends on the particle. If $c_{H} U_{H}^{0}=m c^{2}$, the energy at rest of the particle assumes the same meaning of the other terms, restoring the symmetry lost adding it. The second term, $c_{H} U_{H}$ represents, accordingly, an interaction between the particle and the $\mathscr{H}$ field present in the volume in excess with respect to $H_{0}$. Any volume in the Universe must contain some Higgs field, because its energy cannot be less than zero. Only in certain conditions, e.g. at LHC, the field exceeds the value of $H_{0}$, hence, in most cases, $U_{H}=0$. The third term in the sum is a constant: it can be neglected or kept in the sum, but it is unobservable, since we can only measure differences in energy. The fourth term has the form we expect for the auto-interaction of the field with itself. In those places where there is some excess Higgs field, it contributes to the energy density as all the other fields.

The last term in the sum is proportional to the amount of Higgs field in the volume, through the constant $f_{H} H_{0}$. We can then think at this term as a sort of mass term for the field. The mass term for particles arises because of the interaction of the particles with $H_{0}$. The product $H_{0} H$ can be thought as the interaction of the field $H$ with $H_{0}$, just as $E^{2}$ can be thought as the interaction of the electric field $\mathbf{E}$ with itself.

In ordinary conditions, where $H=0$, the energy of a particle in a volume can then always be written as $\sum_{i} c_{i} U_{i}+L^{3} \sum_{i} \frac{f_{i}}{2} F_{i}^{2}+m c^{2}+C$ where the sums are extended over classical fields and $m c^{2}=c_{H} U_{H}^{0}$ is exactly of the same form of the first addend, while $C$ is a constant that can be neglected because unobservable. This way the meaning of the energy in the volume can be maintained as a measure of the strength of the interactions between particles and fields.

In conditions where $\mathscr{H} \neq H_{0}$, we can interpret the extra terms $c_{H} U_{H}+L^{3} f_{H}\left(\frac{H^{2}}{2}+H_{0} H\right)$ according to different pictures, depending on the scale of the experiment. In a classical picture, the first term represents the interaction of a particle with the field $H$. Note that, since $c_{H} U_{H}^{0}=m c^{2}$, it can be written as $m \frac{c^{2}}{U_{H}^{0}} H$, such that the interaction is proportional to the mass of the particle, as predicted from the Yukawa coupling of the Higgs field to matter fields in particle physics [3]. The term $L 3 \frac{f_{H}}{2} H^{2}$ is completely analogous to the contributions of other fields: the strongest the field, the higher the energy due to auto-interaction. The last term can be interpreted as follows: given a uniform field $H$, the larger the volume, the higher the total field contained in it; the energy contained in the volume is then proportional to the amount of field inside it, as if the field had a rest energy, i.e. a mass. Adopting a quantum mechanics point of view, the field $H$ must be interpreted as a flux of particles. The interaction between Higgs particles and matter particles is still given by $c_{H} U_{H}$, just as the interaction of electrons with photons is represented by $q V$. The term proportional to $H^{2}$ represents the self-coupling of the Higgs particles: they can collide and scatter, for example, just as two photons. The latter term, in fact, represents again a mass term for these particles. In this picture $L^{3} H=L^{3} \alpha N$ where $N$ is the number of Higgs particles in a unit volume. Hence the last term reads $L^{3} f_{H} H_{0} \alpha N$. Given that the coupling with $H_{0}$ for matter fields produces a mass term, the 
interaction of these particles with $H_{0}$ must be of the same nature: such an observation leads to the interpretation of this term as $L^{3} N M c^{2}$, where $M$ is the Higgs boson mass.

\section{Summary}

We presented a simple model in which the need for a Higgs field arises from the observation of a symmetry in the expression of the energy of a particle interacting with a field and of a field interacting with itself. The introduction of the relativistic equivalence between mass and energy breaks the symmetry, that can be recovered postulating the existence of such a field. With the introduction of the new field the energy contained in a volume can be interpreted as a measure of the strength of the interactions between particles and fields contained in it. The model exhibits the same behaviour of the standard model of particle physics and is simple enough to be understood by secondary school students. Moreover, the introduction of such a model allows for a deep and interesting discussion of the physical meaning of the energy.

Our model can easily explain the mass of the vector bosons, too, by a spontaneous symmetry breaking. See [四] for details on this topic.

We look forward to a further development of the model, that can potentially bring some clues on new physics.

\section{References}

[1] Bird, R. B. (1964), 'The Feynman lectures on physics”, Richard P. Feynman, Robert B. Leighton, and Matthew Sands, Addison-Wesley, Reading, Mass, Volume I-II. AIChE J., 10: 794. doi:10.1002/aic.690100602.

[2] Einstein, A. (1905), 'Ist die Trägheit eines Körpers von seinem Energieinhalt abhngig?", Annalen der Physik, 18 639643. doi:10.1002/andp.19053231314.

[3] Steven Weinberg (1967), "A Model of Leptons", Phys. Rev. Lett. 19, 1264. doi:10.1103/PhysRevLett.19.1264.

[4] Giovanni Organtini (2014), "Unveiling the Higgs mechanism to students”, Eur. J. Physics. 33, $1397-1406$. 